\title{
Lidar diagnostics of ionosphere precipitations
}

\author{
Boris Shevtsov ${ }^{1, *}$, Andrey Perezhogin ${ }^{1}$ and Ilya Seredkin ${ }^{1}$ \\ ${ }^{1}$ Institute of Cosmophysical Researches and Radio Wave Propagation FEB RAS
}

\begin{abstract}
Lidar is considered as an electron energy detector of ionosphere precipitations, and the cleaning of radiation belts is like the energy pumping of the ionosphere layer. The excitation efficiency of ionosphere plasma components by precipitations and the features of resonant backscattering of laser radiation in the active ionosphere are discussed. It is shown that in the quantum system in which the lidar plays the role of a master oscillator and the ionosphere layer of the amplifier, different modes of nonlinear oscillations are possible depending on the intensity of the precipitation. Analogies are being made with other natural nonlinear systems.
\end{abstract}

\section{Introduction}

Resonant propagation and back scattering of lidar signals in the exited ionosphere is the example of nature laser system where the lidar is used as master oscillator and the electron precipitations as the energy pumping of ionosphere layers which play the role of spatial distributed resonators and reflectors. The back scattering may be considered as the feedback. On the dependence of ionosphere layer excitations, the different non linear regimes of lidar signal propagation and back scattering will be realized. We will consider lidar sounding in flicker aurora which is induced by electron precipitations from mid-latitude radiation belts (Kamchatka region). Lidar may be used as a detector of electron energy spectrums. The lidar signal is an indicator of the radiation belts cleaning and the ionosphere energy pumping by the charge particle flux which creates the non equilibrium ionosphere layer at $200-600 \mathrm{~km}$ of altitudes. It will be shown what is the effective reflector in the non equilibrium ionosphere layer.

\section{2 lonosphere emission}

The aurora color is defined by the ionosphere content. For the observation of the night sky emission, we used the spectrograph Sp-2500i with CCD Camera PicoStar-HR12 and the telescope with the $60 \mathrm{~cm}$ mirror diameter. Fig. 1 shows the emission spectrums with lines corresponded to the excited atomic hydrogen and nitrogen ions, Tab. 1 (NIST ASD - The National Institute of Standards and Technology, Atomic Spectra Database).

The emission variability is a consequence of a flicker effect. The atomic oxygen and nitrogen ions lines of 558, 561 and $532 \mathrm{~nm}$, especially oxygen, are clearly visible at Fig. 1, while lines of neutral oxygen and nitrogen atoms of 595.858 and $582.954 \mathrm{~nm}$ (NIST ASD) are not observed. It is obvious that neutral atoms are worse excited by electrons than their

\footnotetext{
*e-mail: bshev@ikir.ru
} 


\section{3:00-23:20}

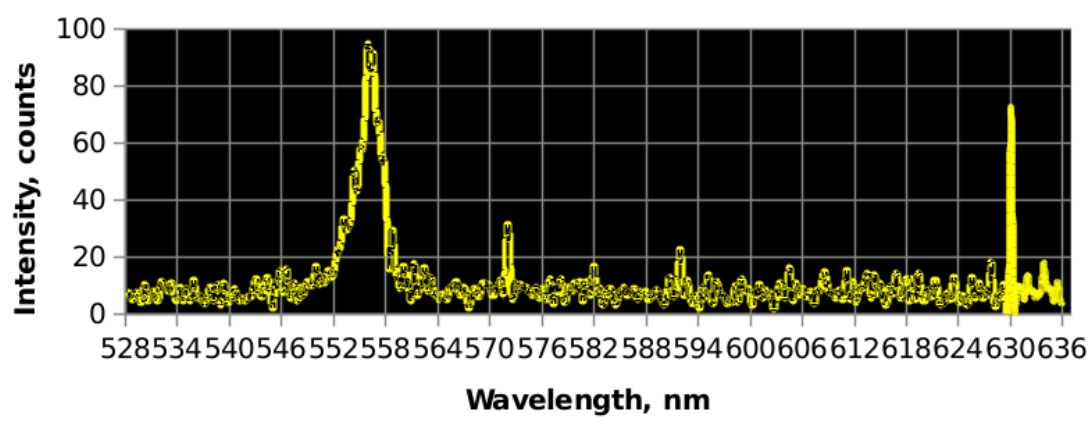

\section{0:30-00:50}

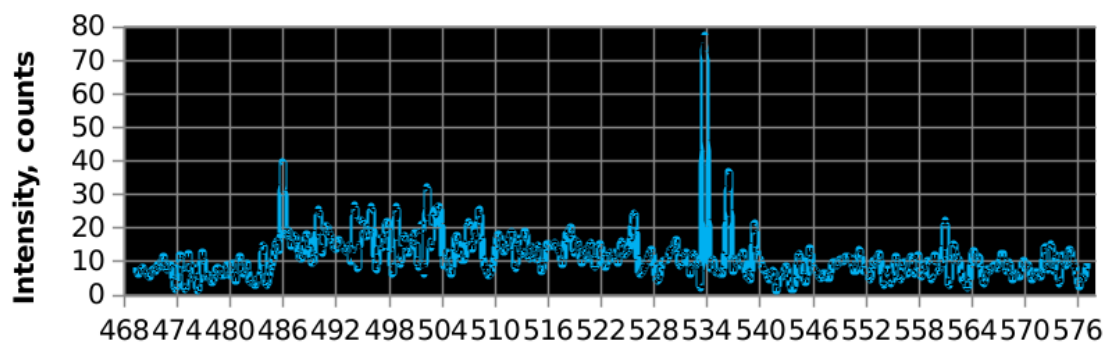

Wavelength, $\mathbf{n m}$

Figure 1. The emission spectrum of precipitations, of 28-29 February 2012, Kamchatka. The signal accumulation times in format (h:m) of local time are shown in upper of pictures.

Table 1. Dipole transitions of excited ions of atomic oxygen and nitrogen (II - singly ionized atom, III - doubly).

\begin{tabular}{|l|l|l|l|l|l|l|l|l|l|}
\hline & $\begin{array}{l}\text { Compo- } \\
\text { nent }\end{array}$ & $\begin{array}{l}\text { Wavelength } \\
\text { Air }(\mathrm{nm})\end{array}$ & $\mathrm{A}_{k i}\left(\mathrm{~s}^{-1}\right)$ & Lower Level & Term & $\mathrm{J}$ & Upper Level & Term & J \\
\hline 1 & OII & 558.3217 & $2,17 \mathrm{e}+06$ & $2 s^{2} 2 p^{2}\left({ }^{1} \mathrm{~S}\right) 3 s$ & ${ }^{2} \mathrm{~S}$ & ${ }^{1} / 2$ & $2 s^{2} 2 p^{2}\left({ }^{3} \mathrm{P}\right) 4 p$ & ${ }^{2} \mathrm{P}^{\circ}$ & ${ }^{3} / 2$ \\
\hline 2 & OII & 561.1072 & $2,14 \mathrm{e}+06$ & $2 s^{2} 2 p^{2}\left({ }^{1} \mathrm{~S}\right) 3 s$ & ${ }^{2} \mathrm{~S}$ & $1 / 2$ & $2 s^{2} 2 p^{2}\left({ }^{3} \mathrm{P}\right) 4 p$ & ${ }^{2} \mathrm{P}^{\circ}$ & $1 / 2$ \\
\hline 3 & NII & 532.0202 & $4.20 \mathrm{e}+07$ & $2 s 2 p^{2}\left({ }^{4} \mathrm{P}\right) 3 p$ & ${ }^{5} \mathrm{P}^{\circ}$ & 2 & $2 \mathrm{~s} 2 \mathrm{p}^{2}\left({ }^{4} \mathrm{P}\right) 3 \mathrm{~d}$ & ${ }^{5} \mathrm{P}^{\circ}$ & 1 \\
\hline 4 & NIII & 532.0870 & $5.68 \mathrm{e}+07$ & $\left.2 \mathrm{~s} 2 \mathrm{p}^{3} \mathrm{P}^{\circ}\right) 3 \mathrm{p}$ & ${ }^{2} \mathrm{D}$ & $5 / 2$ & $2 \mathrm{~s} 2 \mathrm{p}\left({ }^{3} \mathrm{P}^{\circ}\right) 3 \mathrm{~d}$ & ${ }^{2} \mathrm{~F}^{\circ}$ & $7 / 2$ \\
\hline 5 & NII & 532.0958 & $2.52 \mathrm{e}+07$ & $2 \mathrm{~s} 2 \mathrm{p}^{2}\left({ }^{4} \mathrm{P}\right) 3 \mathrm{p}$ & ${ }^{5} \mathrm{P}^{\circ}$ & 1 & $2 \mathrm{~s} 2 \mathrm{p}^{2}\left({ }^{4} \mathrm{P}\right) 3 \mathrm{~d}$ & ${ }^{5} \mathrm{P}$ & 2 \\
\hline
\end{tabular}

ions. The small deviations of the atomic oxygen and nitrogen ion lines (Fig. 1) from their wavelengths (Tab. 1) can be explained by the rough calibration of the spectrograph. This accuracy is sufficient to select the lidar working lines. During emission observations, the electron precipitations were detected by ionosonde.

The aurora altitudes depend on the electrons energy. Our lidar allows to obtained the excited layer altitude distribution [1-4]. There are similar lidars operating at 200-600 km of altitudes and detecting other excited plasma components, N2 [5] and He [6]. For comparison, the ISO has a height about $400 \mathrm{~km}$. 


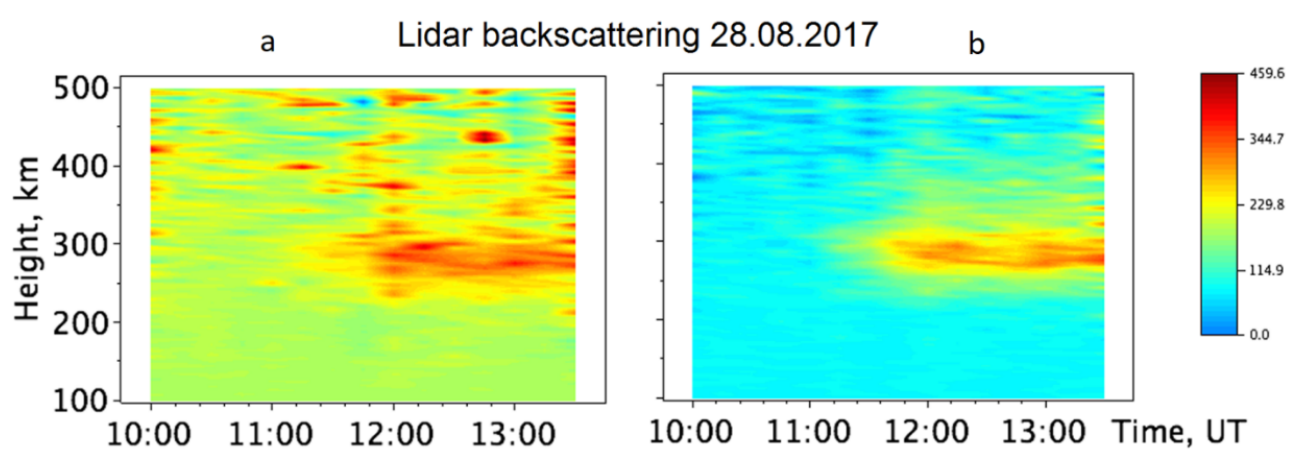

Figure 2. Dynamics of the lidar signal at the wavelengths of $561 \mathrm{~nm}$ (a) and $532 \mathrm{~nm}$ (b). Averaging window of $4.5 \mathrm{~km}$, Local time $+12 \mathrm{~h}$, Color scale: Intensity of lidar signal, counts.

For observations we used two optical generators: dye laser TDL-90 with pumping of Rhodamine 590 by YG-982E and laser Nd: YAG Brilliant-B, for the resonant back scattering on excited atomic ions of oxygen and nitrogen correspondingly. The divergence of the laser beams was about $10^{-5} \mathrm{rad}$.

A telescope with a mirror diameter of $60 \mathrm{~cm}$ and a visual angle of $0.1-1 \mathrm{mrad}$, the divider plate, two interference filters with bandwidth about $1 \mathrm{~nm}$, two photomultipliers Hamamatsu H8259-01 and two Hamamatsu M8784 photon counters were used as a receiver.

The lidar data for two operating lines selected from the Tab. 1 are presented at fig. 2.

The altitude distribution of excited atomic ions of oxygen and nitrogen obtained using two different wavelengths are well correlated that displays the common mechanism of their excitation by a current of charged particles.

The power of the light scattering layer is about $100 \mathrm{~km}$. Earlier it was shown [1-4] that the emergence of this scattering layer correlates with the electron density increasing in the F2 layer (some percents), the increasing in emissions and the appearance of a sporadic $E_{S}$ layer.

The intensities of the scattering signals on the excited atomic ions of oxygen and nitrogen are comparable, while the concentrations of the excited oxygen and nitrogen ions differ approximately by two orders of magnitude. First order is lost to the conversion of the laser frequency, and the second is lost to the low efficiency of oxygen excitation, the oxygen line is narrower than the nitrogen line (Tab. 1). Dynamics of the lidar signal at the wavelengths of $561 \mathrm{~nm}$ and $532 \mathrm{~nm}$ (Fig. 2) show the resonant effect in the back scattering.

\section{Electron energy spectrum}

The altitude distributions of aurora make it possible to estimate the energy spectrum of precipitating charged particles. Fig. 3a shows the lidar signal (1) at $532 \mathrm{~nm}$ and the lidar signal (2) with the geometric factor $(H / 100)^{2}$, were $\mathrm{H}$ is altitude. Fig. $3 \mathrm{~b}$ shows the ionization rate calculated for the Maxwell spectrum of electrons with the average energy of $120 \mathrm{eV}$ (1) and for the mono energetic electrons with the average energy of $330 \mathrm{eV}$ (2). Electron energies were chosen so that the ionization rate maximum corresponds to the height of the signal maximum $(285 \mathrm{~km})$.

The ionization rate profiles (Fig. 3b) were obtained as in [7, 8]. For the calculation, the neutral components $\mathrm{N}_{2}, \mathrm{O}$ and $\mathrm{O}_{2}$ densities were determined from the model NRLMSISE-00. The typical for the aurora zone electron flux $108 \mathrm{~cm}^{-2} \mathrm{~s}^{-1}$ was assumed. From the comparison of Fig. 3a and 3b, we can conclude that the mono energy electrons were injected. 
a 28.08 .2017 11:30-13:30 UT

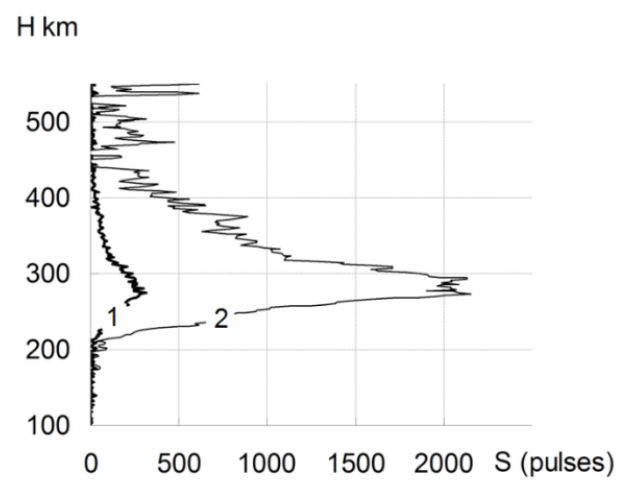

b Calculated ionisation rate

$\mathrm{H} \mathrm{km}$

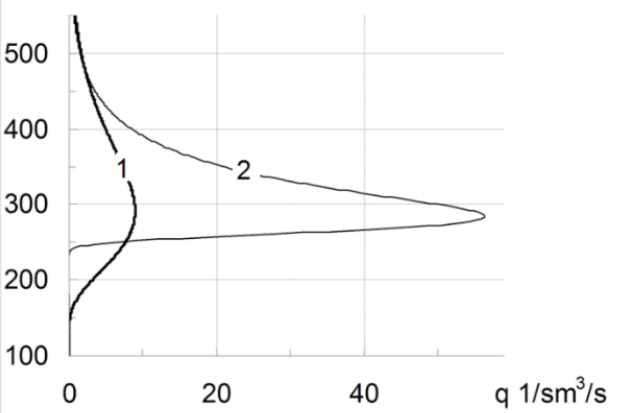

Figure 3. Lidar signal (1) and lidar signal (2) with the geometric factor $(H / 100)^{2}$ at August 28, 2017 (a) and ionization rates (b) for Maxwell spectrum (1) and mono energetic electrons (2).

\section{Equations of back scattering}

Let us consider the back scattering in the exited layer. The result of the paper [9] will be generalized on the case of non linear polarization atoms. The Fourier transformation $R(\tau, \omega)$ of a corn of back scattering operator $R(\tau, t)$ is described by the equation (1) which can be obtained from the system of opposite waves [9].

$$
\frac{\partial}{\partial \tau} R(\tau, \omega)=-2 i k R(\tau, \omega)-i k P(\tau, \omega)(1+R(\tau, \omega)),
$$

where $P(\tau, \omega)$ - Fourier transformation of medium polarization $P(\tau, t), k=\omega / c$ - wave number, $c$ - light velocity and $\tau-$ thickness of the scattering layer. The inverse Fourier transformation of (1) gives the equation for $R(\tau, t)$ :

$$
\frac{\partial}{\partial \chi} R(\chi, t)=-\frac{\partial}{\partial t} R(\chi, t)-\frac{1}{2} \frac{\partial}{\partial t} \int d \zeta P(\chi, t-\zeta)(\delta(\zeta)+R(\chi, \zeta)),
$$

where $\chi=2 \tau / c-$ double travel time in the layer.

In the case of nonlinear polarization atoms [10], $r_{a b}$ - atomic dipole moment matrix and $\sigma_{a b}-$ density matrix.

$$
\dot{\sigma}=-i\left[H_{0}+V, \sigma\right] / \hbar, H_{0}=\left[\begin{array}{cc}
W_{a} & 0 \\
0 & W_{b}
\end{array}\right]
$$

where $W_{a}$ and $W_{b}$ - energy of upper and lower levels, $V(t)=-e r_{a b} E(t)$ - energy of interaction, $E(\chi, t)=\delta(t)+R(\chi, t), \Omega(t)=V(t) / \hbar$ - formally defined Rabi frequency with singular term $\delta(t)$, which specifies the initial conditions for density matrix $\sigma_{0}(\chi)_{t=0}$ which is vector on the Bloch sphere.

The equations (2) and (3) are the Maxwell-Bloch equations which describe the back scattering of the initial wave on the non linear oscillators with initial conditions and under the driving which is realized by the total field $E(\chi, t)$. The layer of ionosphere is a resonator and reflector. The back scattering wave plays the role of feedback. The equation (1) is a spectral presentation of the equation (2). From (1) we see that the back scattering is the sum of additive and multiplicative processes. The last corresponds to multiple back scattering which is negligible for thin layer. 
The value $n(\chi)$ gives $n(\chi) d \chi=2 n(\tau) d \tau / c$, which is the effective thickness of the layer. The initial conditions $\sigma_{0}(\chi)$ (inversion and polarization) at the double spatial harmonics $2 k$ define the back scattering. The harmonic $2 k_{0}=2 \sigma_{0} / c$, where $\omega_{0}=\left(W_{a}-W_{b}\right) / \hbar$ is the resonant frequency, gives the resonant back scattering which principal differs from the resonant absorption and fluorescence.

Note, that $n(\chi)$ and $\sigma_{0}(\chi)$ are the random functions which are make ready by electron precipitations. The charged particles current is responsible the energy pumping of two levels system as in the gas laser. We will be assume that stochastic $n(\chi)$ and $\sigma_{0}(\chi)$ are ready for lidar sounding. The inversion cases \pm 1 and the double spatial harmonics $2 k_{0}$ (Brag's condition) are more interesting.

In resonant propagation and scattering, the $\pi$-impulse plays the role of unit pulse. The wave source $U_{0}(t)$ is located on the bottom edge $\tau$ of the layer. Amplitude, frequency content and duration of $U_{0}(t)$ have to correspond to the composition of $\pi$-impulses. The total wave on the bottom edge of the layer is $E(\chi, t)=U_{0}(t)+\int d \zeta R(\chi, t-\zeta) U_{0}(\zeta)$. The wave in the layer may be obtained from equation

$$
\left(\frac{\partial}{\partial z}-\frac{\partial}{\partial t}\right) U(z, t)=\frac{\partial}{\partial t} P(z, t)
$$

with boundary condition $U_{0}(t)$ on the bottom edge of the layer.

The Maxwell-Bloch equations (1-3) describe the non linear polarization response of the Delta impulse. The equations (1-2) give the back scattering (reflection) and ( (3)) - a transmitted wave. The Delta impulse result defines the response of the arbitrary shape pulse. The linear polarization of the weak pulse was considered in [10].

\section{Conclusion}

Electron current of precipitations changes weakly the ionization and emission. The current excites additionally the existing ions and creates the condition for resonant propagation and back scattering. There are not enhancement emission and resonant back scattering of neutrals. The resonant emission, absorption and back scattering of ions play the major roles. The density of two level atoms and the non linear polarization provide the back scattering.

\section{References}

[1] V.V. Bychkov, Y.A. Nepomnyashchiy, A.S. Perezhogin, B.M. Shevtsov, Earth, Planets and Space 66, 150 (2014)

[2] V.V. Bychkov, Y.A. Nepomnyashchiy, A.S. Perezhogin, B.M. Shevtsov, V.N. Marichev, Optics of the atmosphere and the ocean 30, 209 (2017)

[3] V.V. Bychkov, A.S. Perezhogin, I.N. Seredkin, B.M. Shevtsov, On the role of the method of measuring the background signal in the lidar measurements of the upper atmosphere, in 23rd International Symposium on Atmospheric and Ocean Optics: Atmospheric Physics (International Society for Optics and Photonics, 2017), Vol. 10466, p. 1046677

[4] V.V. Bychkov, Y.A. Nepomnyashchiy, A.S. Perezhogin, B.M. Shevtsov, History of Science and Technology 8, 85 (2017)

[5] R. Collins, J. Li, C. Martus, Geophysical Research Letters 42, 665 (2015)

[6] L. Waldrop, R. Kerr, S. Gonzalez, M. Sulzer, J. Noto, F. Kamalabadi, Journal of Geophysical Research: Space Physics 110 (2005) 
[7] M. Deminov, V. Khegai, Geomagnetism and Aeronomy 20, 145 (1980)

[8] M. Deminov, Plasma Heliogeophysics 20, 145 (1980)

[9] A. Perezhogin, B. Shevtsov, Journal of Communications Technology and Electronics 59, 40 (2014)

[10] R.J. Glauber, Quantum optics, in Quantum Optics (1969) 